\section{FITTRW: A parame ter estimation program for a general random walk model analysis of two-choice response time (2CRT) data}

\section{RICHARD A. HEATH \\ University of Newcastle, New South Wales 2308, Australia}

FITTRW is a FORTRAN program designed to estimate parameters of a general class of random walk models for two-choice response time (2CRT) tasks. Such tasks involve the presentation of two stimuli, Sa and $\mathrm{Sb}$, for which the appropriate response is $\mathrm{Ra}$ and $\mathrm{Rb}$, respectively. The models that can be fit include relative judgment theory (RJT), devised by Link and Heath (1975), the model of Laming (1968), which includes starting point variability in the random walk, and the extensions of the random walk model that cater to nonstationarities generated by brief stimulus presentations (Heath, 1981, 1982a, 1982b). In this model, referred to as the tandem random walk (TRW) model, the random walk decision process is represented by two stages with differing drift rates. In a special version of the TRW model applicable when the stimulus duration is brief and accuracy is emphasized, the second, or terminal, stage has zero drift

FITTRW uses the STEPIT function minimization routine (Chandler, Note 1), which searches a predefined parameter space for a parameter vector that minimizes an objective function such as the mean sum of squared errors (MSe), or chi square if standard errors of the mean RTs are included. The program requires response proportion and mean RT data for both correct and erroneous responses.

Input. The data are entered by means of an assigned input file (normally on disk) and user responses to program prompts. The first two lines of the data file contain a description of the data set and the number of experimental conditions. This is followed by the number of lcvels of each of the following parameters: $A=$ the response threshold or caution, the distance the random walk has to travel from the origin in order for a decision to be made. $\mathrm{C}=$ the response bias as represented by the mean starting point of the random walk. $U A=$ the mean step size, or drift, of the random walk generated by Sa. $\mathrm{UB}=$ the drift of the random walk generated by $\mathrm{Sb}$. $\mathrm{VOA}=$ the starting point variance for $\mathrm{Sa} . \mathrm{VOB}=$ the starting point variance for $\mathrm{Sb} . \mathrm{V} 1 \mathrm{~A}=$ the variance of random walk steps for Sa. V1B = the variance of random walk steps for $\mathrm{Sb}$. RTA = the residual mean RT for

I wish to thank R. Millward, Director of the Cognitive Science Center at Brown University, for the generous use of the center's VAX-11/780 computer. I also wish to acknowledge the use of the STEPIT subroutine written by J. P. Chandler, Computer Science Department, Oklahoma State University, Stillwater, Oklahoma 74074.
$\mathrm{Ra} . \mathrm{RTB}=$ the residual mean $\mathrm{RT}$ for $\mathrm{Rb} . \mathrm{COA}=$ the mean displacement of the starting point of the terminal stage of the TRW model for $\mathrm{Sa}$. $\mathrm{COB}=$ the mean dis placement of the starting point of the terminal stage of the TRW model for $\mathrm{Sb}, \mathrm{GA}=$ the moment generating function (mgf) asymmetry parameter of RJT for Sa. $\mathrm{GB}=$ the mgf asymmetry parameter of RJT for $\mathrm{Sb}$

The program allows symmetric RT models such as RJT (Link, 1975) to be fit by constraining the drift parameters, UA and UB, to be equal but opposite in sign. Single-condition experiments such as "samedifferent" judgments that generate asymmetric drifts can also be accommodated.

For each parameter specified, the initial, maximum, and minimum values and the starting and ending step sizes for the STEPIT routine are entered. Parameters can be fixed for a given run of the program to provide sensitivity tests. Then follow the data for each experimental condition and a classification of each condition in terms of levels of the parameters suitable for the model under test.

Before execution, the program prompts the user for information on (1) whether the data arise from a symmetric or a single-condition experiment, such as a samedifferent judgment task, (2) whether each level of the parameters has the same starting value (recommended for ease of use of the program), (3) whether a terminal zero-drift TRW model is being fit, (4) whether standard errors of mean RTs are available, (5) whether a trace of the convergence history for STEPIT is required, and (6) the maximum number of function calls to STEPIT.

Output. The output file generated by FITTRW contains (1) the starting values for the STEPIT routine, (2) the parameter estimates and their standard errors, (3) the correlation matrix for the parameter estimates, (4) the number of function computations and the final value of MSE, and (5) a table of parameter estimates and the observed and predicted response proportions and mean RTs for each experimental condition.

Computer and Language. Program FITTRW is written in VAX-11 FORTRAN and runs on a VAX-11/780 computer using the VMS operating system. No library routines are required.

Restrictions. The program can handle up to 50 parameters, but this value can be altered by changing the dimensions of arrays in FITTRW and STEPIT. The program requires $41 \mathrm{~KB}$ of memory space on a VAX$11 / 780$, and the average execution time, which depends on the maximum number of function evaluations and the minimum parameter increment, is a few minutes of CPU time.

Availability. A source listing, users' guide, and sample input and output can be obtained free of charge by writing to Richard A. Heath, Department of Psychology, 
University of Newcastle, New South Wales 2308, Australia. This information can be obtained on 8-in. floppy disk in RT-11 format by sending a blank floppy disk or $\$ 20$ to cover costs.

\section{REFERENCE NOTE}

1. Chandler, J. P. Subroutine STEPIT (Quantum Chemistry Program Exchange No. 307). Oklahoma State University, June 1975.

\section{REFERENCES}

HEATH, R. A. A tandem random walk model for psychological discrimination. British Journal of Mathematical and Statistical Psychology, 1981, 34, 76-92.

Heath, R. A. Memory for the order of sequential visual events. Australian Journal of Psychology, 1982, 34, 267-279. (a)

Heath, R. A. A model for signal detection based on an adaptive filter. Biological Cybernetics, 1982, 45, 95-100. (b)

Laming, D. R. J. Information theory of choice-reaction times. London: Academic Press, 1968.

LiNk, S. W. The relative judgment theory of two choice response time. Journal of Mathematical Psychology, 1975, 12, 114-135.

Link, S. W., \& Heath, R. A. A sequential theory of psychological discrimination. Psychometrika, 1975, 40, 77-105.

(Accepted for publication December 30, 1982.) 